\title{
Strong Coupling of Exciton-Polaritons in a Bulk GaN Planar Waveguide: Quantifying the Coupling Strength
}

\author{
C. Brimont,${ }^{1}$ L. Doyennette, ${ }^{1}$ G. Kreyder, ${ }^{2}$ F. Réveret, ${ }^{2}$ P. Disseix, ${ }^{2}$ F. Médard $\odot,{ }^{2}$ \\ J. Leymarie, ${ }^{2}$ E. Cambril,${ }^{3}$ S. Bouchoule, ${ }^{3}$ M. Gromovyi, ${ }^{3,4}$ B. Alloing, ${ }^{4}$ S. Rennesson, ${ }^{4}$ F. Semond, ${ }^{4}$ \\ J. Zúñiga-Pérez, ${ }^{4}$ and T. Guillet $\oplus^{1,5, *}$ \\ ${ }^{1}$ Laboratoire Charles Coulomb (L2C), Université de Montpellier, CNRS, Montpellier, France \\ ${ }^{2}$ Université Clermont Auvergne, CNRS, SIGMA Clermont, Institut Pascal, Clermont-Ferrand, France \\ ${ }^{3}$ Centre de Nanosciences et de Nanotechnologies, CNRS, Université Paris-Saclay, France \\ ${ }^{4} U C A, C R H E A-C N R S$, Rue Bernard Gregory, 06560 Valbonne, France \\ ${ }^{5}$ Department of Physics, SUPA, University of Strathclyde, Glasgow, United Kingdom
}

(Received 13 February 2020; revised 21 September 2020; accepted 20 October 2020; published 23 November 2020)

\begin{abstract}
We investigate the demonstration and quantification of the strong coupling between excitons and guided photons in a GaN slab waveguide. The dispersions of waveguide polaritons are measured from $T=6$ to $300 \mathrm{~K}$ through gratings. They are carefully analyzed within four models based on different assumptions, in order to assess the strong-coupling regime. We prove that the guided photons and excitons are strongly coupled at all investigated temperatures, with a small (11\%) dependence on the temperature. However, the values of the Rabi splitting strongly vary among the four models: the "coupled oscillator" model overestimates the coupling; the analytical "Elliott-Tanguy" model precisely describes the dielectric susceptibility of $\mathrm{GaN}$ near the excitonic transition, leading to a Rabi splitting equal to $82 \pm 10 \mathrm{meV}$ for fundamental transverse-electric mode; the experimental ellipsometry-based model leads to smaller values of $55 \pm 6$ meV. We evidence that, for waveguides including active layers with large oscillator strengths, as required for room-temperature polaritonic devices, a strong bending of the modes' dispersion is not necessarily the signature of the strong coupling, which requires for its reliable assessment a precise analysis of the material dielectric susceptibility.
\end{abstract}

DOI: 10.1103/PhysRevApplied.14.054060

\section{INTRODUCTION}

Exciton-polaritons are the dressed states of semiconductor excitons when they interact with photons in the strong-coupling regime. Compared to photons, the interactions between polaritons are enhanced by a few orders of magnitude [1,2], allowing for the exploration of a wide range of collective quantum states and phenomena [3-6] inspired from the physics of cold atoms. Most of these studies have been performed on microcavity-polaritons, where two-dimensional (2D) photons are confined in a vertical Fabry-Perot cavity [7]. More complex polariton states can be engineered through photonic circuits [8-10] or optical control of the excitation pattern $[5,11]$. Alternative geometries have recently been explored, where excitons are coupled to Tamm plasmons [12,13], Bloch surface states [14], micro- and nanowires [15-18], and guided modes in a slab [19].

*thierry.guillet@umontpellier.fr
The waveguide geometry provides an interesting framework for polariton fluids propagating with a large group velocity, and with a strong nonlinearity, leading to the formation of bright or dark solitons carrying a very low energy per pulse of about one picojoule in GaAsbased waveguides at cryogenic temperatures [20,21], and pulse amplitude modulation of about $100 \mathrm{pJ}$ in GaNbased waveguides at room temperature [22]. The waveguide geometry was recently investigated in $\mathrm{GaN}$ and $\mathrm{ZnO}$ semiconductors in order to achieve large Rabi splittings [23-26], attaining up to about $200 \mathrm{meV}$ for a $\mathrm{ZnO}$ slab waveguide and enabling the demonstration of a polariton laser and a polariton amplifier operating up to room temperature [26]. Indeed, room-temperature polaritonics requires robust polaritons involving excitons with a large oscillator strength and a large binding energy, found either in wide bandgap semiconductors or in organic materials [27]. When large Rabi splittings are achieved in these systems, a proper measurement and modeling of the dispersion of the eigenmodes is necessary, a much more difficult task than when dealing with standard semiconductors (e.g., GaAs) and a vertical geometry [25,28]. Indeed, on 
the experimental side, the damping of the upper polariton branch by the absorption of continuum states frequently precludes its observation and the direct measurement of the Rabi splitting between lower and upper polariton states $[29,30]$; on the theoretical side, refractive index contributions from bound excitons, unbound scattering states, and continuum states are a long-debated issue [31,32], which becomes prominent when investigating wide bandgap semiconductors and organic materials [33]. This is due to the large corrections they introduce to the so-called background refractive index. The role of these contributions was already evidenced in the dispersion of polaritons in vertical microcavities based on wide bandgap materials [27,34].

In general, the precise determination of the excitonphoton coupling strength requires the identification of the condition of zero detuning, where the energies of the bare photon mode (without coupling to the excitons) and of the exciton are equal. Extracting such a simple condition from the experimental dispersions becomes a challenging task due to the difficulty to answer the question: what would be the dispersion of a bare waveguide mode in a gedanken experiment where exciton oscillators would be "turned off" in the optical response of the active layer? To answer this question, we measure the polariton dispersions in a bulk GaN polariton waveguide from low to room temperature and propose four models to simulate them, extracting therefrom the modeldependent exciton-photon coupling strengths. These models go from the most basic and most-frequently employed coupled-oscillator models to more elaborate ones that implement more accurate descriptions of the materials' dielectric susceptibility. We compare and analyze critically the very different estimates of the exciton-photon coupling strength while fitting the same experimental polariton dispersions.

\section{SAMPLE}

The structure schematically depicted in Fig. 1(a) has been grown by molecular beam epitaxy on a Si(111) substrate. The epitaxy of a thick $(470 \mathrm{~nm})$ AlN buffer at high temperature $\left(1000^{\circ} \mathrm{C}\right)$ is followed by a 170 -nm-thick $\mathrm{Al}_{0.65} \mathrm{Ga}_{0.35} \mathrm{~N}$, before depositing the $\mathrm{GaN}$ waveguide core. The AlN and $\mathrm{Al}_{0.65} \mathrm{Ga}_{0.35} \mathrm{~N}$ cladding layers prevent the overlap between the waveguide photonic mode and the Si substrate, which would otherwise result in a strong absorption. The topmost surface of the GaN waveguide displays a surface roughness as low as $1 \mathrm{~nm} \mathrm{rms}$, as illustrated in Fig. 1(b). The typical thickness of the core GaN layer, measured in a scanning electron micrograph (SEM), is $100 \mathrm{~nm}$. The sample is almost crack-free, with a typical distance between cracks of the order of several millimeters.

First-order grating couplers have been defined by electron beam lithography [Fig. 1(c)] using a negative-tone hydrogen silsesquioxane (HSQ, flowable oxide) resist. After HSQ resist development, the sample is baked under an $\mathrm{O}_{2}$ atmosphere at $440^{\circ} \mathrm{C}$ for $1 \mathrm{~h}$ to stabilize the $\mathrm{HSQ}$ refractive index to a value of $n \approx 1.43$ and reduce the extinction coefficient to $k<10^{-4}$ around $\lambda=365 \mathrm{~nm}$. The HSQ thickness is about $80 \mathrm{~nm}$ after thermal cure. Each grating spans over a $100 \times 100 \mu \mathrm{m}^{2}$ area, with the grating grooves oriented parallel to the direction $\langle 1 \overline{1} 00\rangle$.

Such a grating couples a wave propagating with a propagation constant $\beta$ parallel to the direction $\langle 11 \overline{2} 0\rangle$ and an energy $E=\hbar \omega$ to an outcoupled light wave at an angle $\theta$ according to the first-order diffraction condition

$$
\beta=\frac{\omega}{c} \sin (\theta) \pm \frac{2 \pi}{\Lambda} .
$$

The grating period $\Lambda$ has been chosen for an optimal outcoupling perpendicular to the sample surface in the zerodetuning regime [for the fundamental transverse-electric
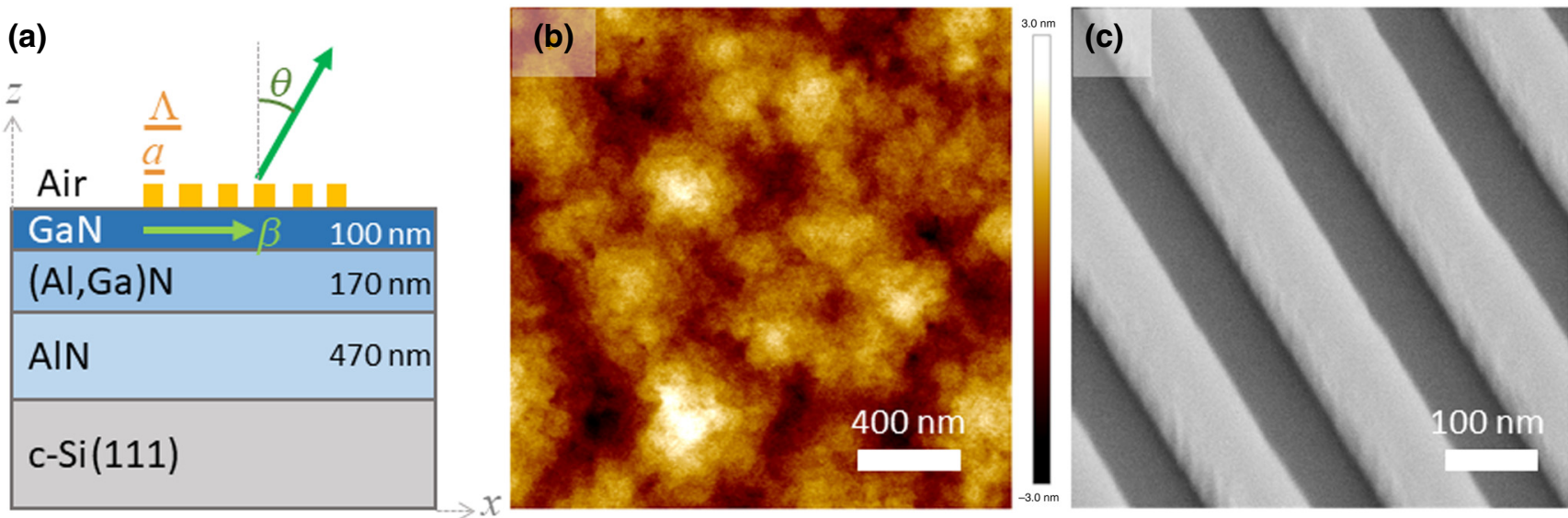

FIG. 1. (a) Sample structure; $\beta$ represents the propagation constant of the guided polaritons, and $\theta$ is the outcoupling angle. (b) Atomic force microscopy (AFM) image of the top GaN surface (z scale $6 \mathrm{~nm}$; rms roughness $0.8 \mathrm{~nm}$ ). (c) SEM of the etched diffraction grating. 
(TE0) mode]. A series of gratings with varying periods from 140 to $170 \mathrm{~nm}$ by steps of $6 \mathrm{~nm}$ are fabricated, all of them with a measured fill factor $a / \Lambda=0.43 \pm 0.01$, where $a$ is the width of each grating stripe.

The TE0 [respectively the fundamental transversemagnetic (TM0) mode] modal confinement factor is estimated to be of $80 \%$ (respectively $65 \%$ ) in the GaN guiding core while the overlapping with the grating is typically lower than $3 \%$, leading to a relatively weak extraction loss. The combination of a surface roughness of the order of 1 $\mathrm{nm}$ and a surface morphology made up of step-and-terrace mounds with a diameter of the order of $1 \mu \mathrm{m}$ or less, results in propagation losses due to surface scattering [35] estimated to $8 \mathrm{~cm}^{-1}$ at $\lambda=370 \mathrm{~nm}$. Thus, this source of loss should only dominate the absorption losses at strongly negative detuning.

\section{EXPERIMENTAL POLARITON DISPERSIONS}

The polariton dispersions are measured through angleresolved microphotoluminescence under pulsed nonresonant excitation (400 ps pulse width, $4 \mathrm{kHz}$ repetition rate, at $266 \mathrm{~nm}$; approximately $30 \mu \mathrm{m}$ defocused excitation spot). The pulsed excitation allows for an efficient relaxation of the exciton reservoir towards the polariton branch. The excitation density (typically $1 \mathrm{~mJ} \mathrm{~cm}^{-2}$ per pulse) is comparable to the threshold for previously investigated polariton lasers based on $\mathrm{GaN}$ microcavities [36-39], even though lasing is not observed here due to the absence of any cavity for the guided polaritons. It is also comparable to the threshold of the recently demonstrated $\mathrm{ZnO}$ waveguide polariton laser [26].

The photoluminescence is collected in a Fourier imaging scheme: the back-focal plane of the microscope objective (OFR LMU-20x, numerical aperture 0.40) is imaged onto the entrance slit of the imaging spectrometer and spectrally dispersed $(0.1 \mathrm{~nm}$ resolution). The angle-resolved photoluminescence measured at $T=6 \mathrm{~K}$ for a grating period $\Lambda=152 \mathrm{~nm}$ is presented in Fig. 2(a). We observe two sets of dispersion curves for positive and negative values of the wavevector $\beta$ along the $z$ propagation axis, i.e., left- and right-propagating polaritons: $\beta=E \sin (\theta) / h c \pm$ $2 \pi / \Lambda$ with positive and negative dispersion slopes $E(\theta)$. The broad emission line around $3.5 \mathrm{eV}$ does not present any structure in Fourier space; it is attributed to localized and donor-bound excitons.

In order to cover a broader range of wavevectors, we present in Fig. 2(b) the dispersions measured as a function of $\beta$ for grating periods ranging from 152 to $170 \mathrm{~nm}$. The identification of the TE and TM modes is deduced from polarization-resolved measurements. Let us underline that the upper polariton branch, above the exciton energy, is not observed in the experiments due to its strong damping by the absorption of the electron-hole band-to-band
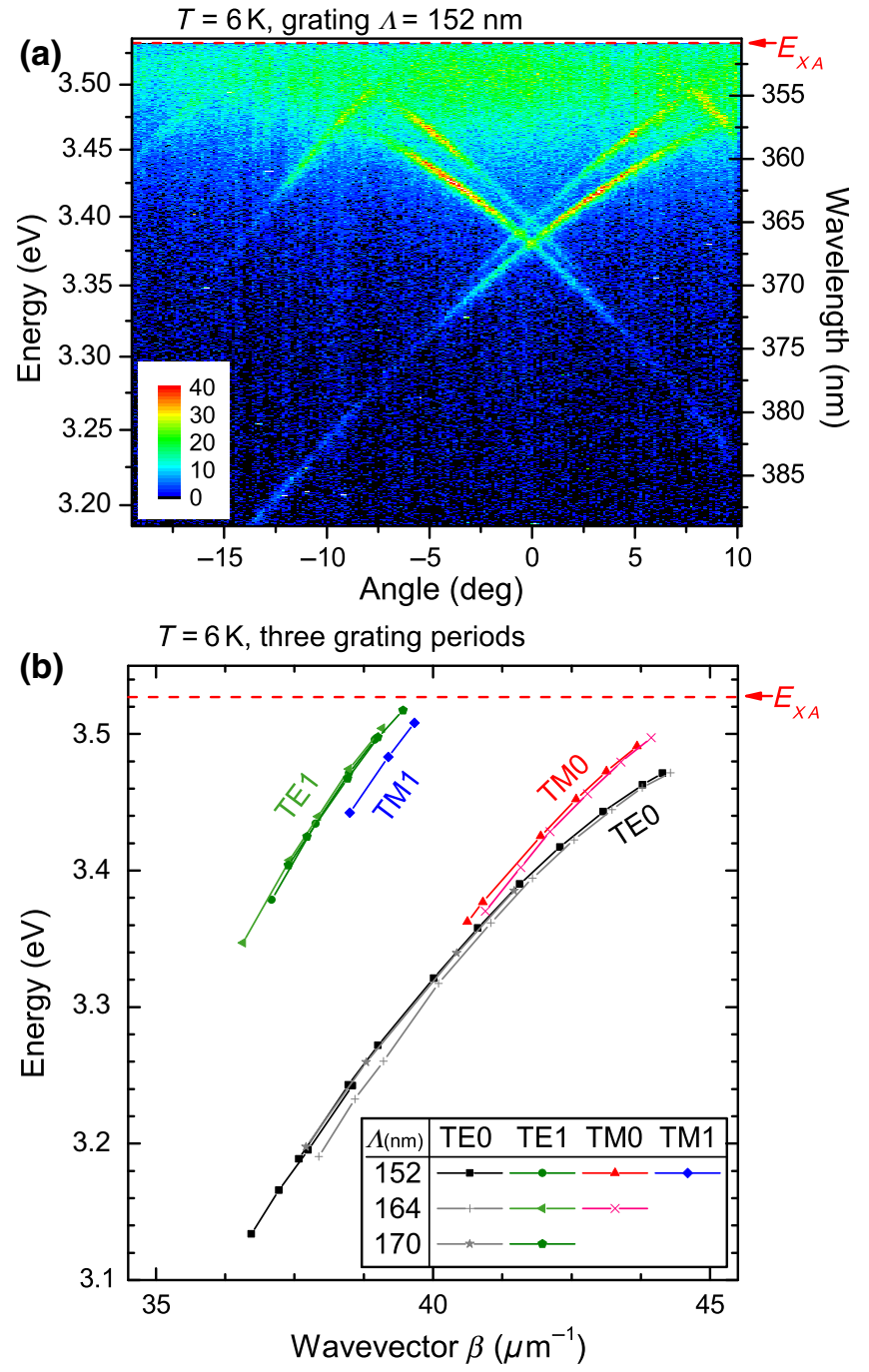

FIG. 2. (a) Angle-resolved photoluminescence $(T=6 \mathrm{~K})$ of the $\mathrm{GaN}$ waveguide diffracted at the grating (period $\Lambda=$ $152 \mathrm{~nm}$ ). (b) Experimental exciton-polariton dispersions of the lower polariton branch extracted from three diffraction gratings, whose periods are indicated in the inset table.

transitions, as discussed earlier for $\mathrm{GaN}$ and $\mathrm{ZnO}$ microcavities $[29,40]$. The observed bending of the TE0 and TM0 dispersions when approaching the exciton energy is expected to be the signature of the strong-coupling regime between the guided photon and the exciton modes, as will be confirmed later in the discussion. The next section is devoted to the quantitative analysis of these dispersions. Note that the TE1 and TM1 modes, which are mostly confined in the cladding layers, show a much smaller bending.

\section{MODELING THE POLARITON DISPERSION}

As discussed in a seminal work on polaritons in microcavities [41], the coupled oscillator model provides a simple implementation of the quantum theory of the 
exciton-photon interaction, leading to two coupled modes separated by the Rabi splitting. On the other side, the semiclassical theory is based on a calculation of the optical response through the resolution of the Maxwell equations with a nonlocal linear susceptibility of the active medium. Both approaches provide a complementary understanding of the strong coupling between excitons and photons. In the case of microcavities, the predicted splitting between polariton resonances depends on the probed optical response (reflectivity, transmission, photoluminescence) and it is comparable but not equal to the Rabi splitting deduced from quantum theory [41].

In the iconic case of a single GaAs quantum well in a microcavity, the simple "Lorentzian excitonic" susceptibility provides a relevant description of the active layer, even though all nonresonant contributions are approximated in a frequency-independent background dielectric constant [41]. This "Lorentzian excitonic" model is implemented in most transfer-matrix simulations of organic and inorganic semiconductor microcavities.

In this section the analysis of the measured polariton dispersions is investigated through four different approaches. The two first models are commonly used in the polaritonics literature to establish the strong-coupling regime in vertical microcavities: the coupled oscillator model (model $A$ ) on one side, and the semiclassical approach with a "Lorentzian excitonic" susceptibility (model $B$ ) on the other side. The discrepancy between the measured dispersions and these two standard models $A$ and $B$ lead us to consider two more elaborate dielectric susceptibilities for the GaN active layer: the so-called "Elliott-Tanguy" model (model $C$ ) includes all contributions from bound and unbound excitonic states in the dielectric susceptibility; meanwhile, the "empirical" model (model $D$ ) implements the respective contributions from bound excitonic transitions and from all other transitions in the susceptibility as deduced from spectroscopic ellipsometry measurements performed at room temperature $[42,43]$. The fitting of the polariton dispersions with each of the four models will be performed from $T=6 \mathrm{~K}$ (Sec. IV) to $T=300 \mathrm{~K}$ (Sec. VI), while the accuracy of the temperature-dependent susceptibility of each model will be tested by comparing it to the experimental ellipsometry measurements performed at $T=300 \mathrm{~K}$. Moreover, the TE and TM modes calculated within isotropic and anisotropic susceptibilities will be further compared. Finally, we contrast the four interpretive frameworks and conclude by discussing the reliability and accuracy of each.

\section{A. Coupled oscillator model $(\operatorname{model} \boldsymbol{A})$}

Let us first compare the measured dispersions of the TE0 and TM0 modes with the coupled oscillator model. As discussed in Appendix A, the two optical polarizations can be treated independently. Model $A$ and model $B$ excitons couple to the bare TE0 mode; since they are closely lying,

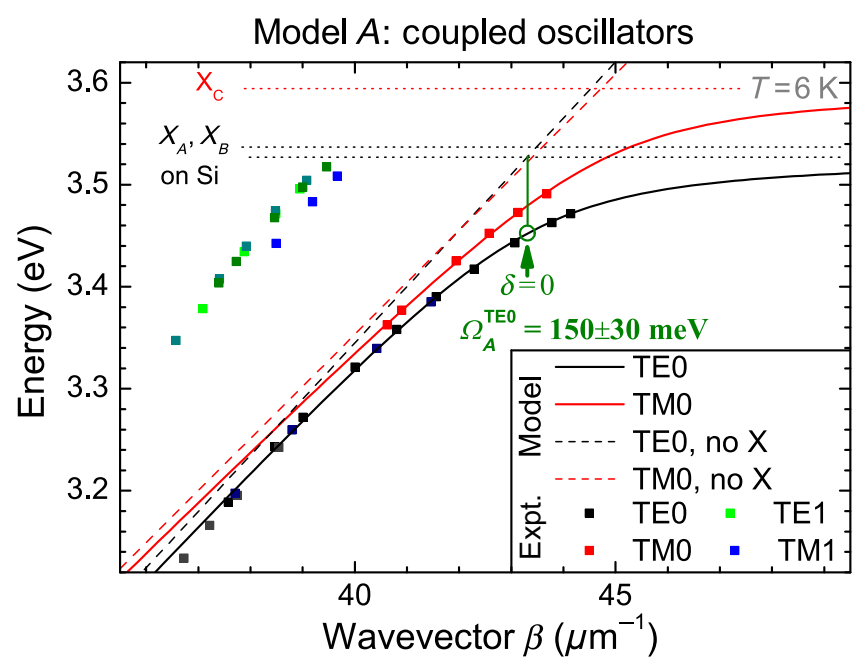

FIG. 3. Model $A$ : coupled oscillator modeling (plain lines) of the TE0 (black) and TM0 (red) polariton dispersions; the experimental dispersions are shown as squares. The corresponding bare waveguide modes are indicated as dashed lines, and the exciton energies as horizontal dotted lines. The green open circle indicates the zero-detuning condition, and the green vertical segment represents half of the exciton-photon coupling strength.

with a splitting much smaller than the expected Rabi splitting, we consider them as a single excitonic oscillator. The $C$ exciton couples to the bare TM0 mode. For simplicity, we do not consider here the coupling of the excitons to higher-order optical modes (TE1, TM1, and beyond). For each polarization, the coupling is therefore modeled by a wavevector-dependent $2 \times 2$ Hamiltonian. Such an approach can be generalized to a broader set of excitonic and photonic modes [28].

We assume a linear dispersion of the bare photon mode within the spectral range of interest. The exciton energies are fixed from reflectivity measurements (see Appendix A). Both calculated TE0 and TM0 polariton dispersions are shown in Fig. 3. The fit over three parameters (the two coefficients of the photon line and the exciton-photon coupling strength) provides an estimate of the excitonphoton coupling strength for the TE0 polariton mode: $\Omega_{A}^{\mathrm{TE} 0}=150 \pm 30 \mathrm{meV}$. The large uncertainty in $\Omega_{A}$ results from the large uncertainty in the group velocity of the bare photon mode, thereby influencing the wavevector $\beta_{\delta 0}^{A}=$ $43.3 \pm 1 \mu \mathrm{m}^{-1}$ that corresponds to zero detuning. The same fitting procedure for the TM0 polariton mode leads to $\Omega_{A}^{\mathrm{TM} 0}=150 \pm 50 \mathrm{meV}$, the uncertainty being even larger than for the TE0 mode because we do not access large negative detunings in the angle-resolved PL experiments.

\section{B. Waveguide modeling with a susceptibility based on Lorentzian excitonic resonances $(\operatorname{model} B)$}

The standard approach when investigating the strong coupling in a vertical microcavity consists in comparing 
the previous coupled oscillator model to transfer matrix simulations [41], in which the photonic modes of the microcavity and the dielectric susceptibility of the exciton active layer (see Ref. [30] for GaN microcavities) are both taken into account. The equivalent for the waveguide geometry is the resolution of the guided modes in a dielectric slab. Here we choose the resolution for the scalar description of the electromagnetic field either in the TE or in the TM polarization, therefore neglecting any TETM coupling. The modes are calculated with $\mathrm{CamFR}^{\circledR}$, an open-source code implementing the vectorial eigenmode expansion method [44], under the approximation of decoupled TE and TM eigenmodes (see Appendix A). This is fully valid for the TE modes in the absence of coupling between $A, B$, and $C$ excitons. Meanwhile, this is an approximation for the TM modes, since the electric field of the TM modes may have a small component along the sample plane, therefore coupling to the $A$ and $B$ excitons. This is also an approximation for the TE mode if we take into account the mixing of oscillator strengths of the $A, B$, and $C$ excitons, but this mixing is of the order of $1 \%$, as discussed in Appendix A. We can therefore consider that the scalar resolution of the waveguide eigenmodes is a relevant approximation.

Following this approach with the effective background dielectric constant as a free parameter, and considering a typical oscillator strength of $40000 \mathrm{meV}^{2}$ for each $A$ and $B$ excitons and $80000 \mathrm{meV}^{2}$ for the $C$ exciton [43,45-48], we compare the calculated and experimental polariton dispersions in Fig. 4(c). It is seen that the calculated polariton dispersion fails to reproduce the experimental measurement: (i) the experimental dispersion near the anticrossing is not reproduced by the model; (ii) the dispersion at negative detunings is not fitted properly, and the corresponding group velocity of the bare photon mode is $20 \%$ larger than the measured one, even if we account for the dispersive refractive index of the $(\mathrm{Al}, \mathrm{Ga}) \mathrm{N}$ and AlN cladding layers. The Rabi splitting extracted from this model is only $97 \pm 10 \mathrm{meV}$, much smaller than the result of the coupled oscillator model.

To unravel the origin of the discrepancy between the experimental and calculated dispersions, for a thick relaxed GaN layer at room temperature, we compare the experimental complex dielectric susceptibility $\varepsilon=\varepsilon_{1}+$ $i \varepsilon_{2}$ to the "Lorentzian" one [Figs. 4(a) and 4(b)]. The model susceptibility is chosen as the sum of three contributions: (i) the Sellmeier dispersive susceptibility associated to the deep $\mathrm{UV}$ pole of $\mathrm{GaN}$, at about $7 \mathrm{eV}[49,50]$ (yellow curve); (ii) an empirical rigid shift of $\varepsilon_{1}$; (iii) the contribution of the excitonic Lorentzian oscillator to the dielectric susceptibility (solid red curve). The calculated susceptibility of the GaN material in the absence of the excitonic resonance is the sum of the two first contributions (dashed red line), and is used to calculate the bare guided mode dispersion in Fig. 4(c).
Model B: Lorentzian oscillator for $X_{1 \mathrm{~s}}$
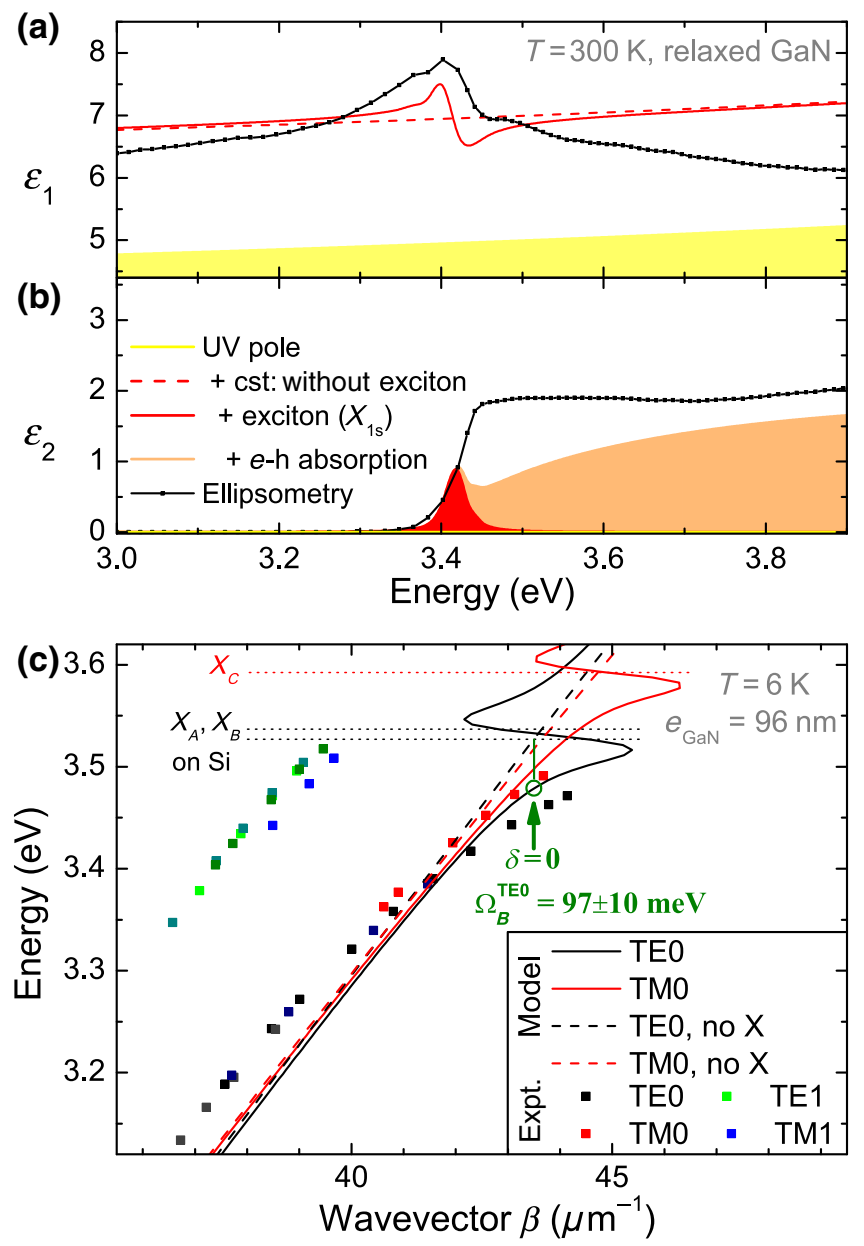

FIG. 4. Model $B$ based on the nonlocal susceptibility of a single Lorentzian excitonic resonance. (a) Real part and (b) imaginary part of the GaN dielectric susceptibility (in-plane light polarization, TE mode, $T=300 \mathrm{~K}$ ): black square, solid lines represent ellipsometry measurements; (red) single Lorentzian oscillator accounting for both $A$ and $B$ excitonic transitions [including a deep UV Sellmeier pole and a constant shift $(+$ cst)]; (orange) electron-hole band-to-band (e-h) contribution to the absorption. (c) Calculated polariton dispersions (solid lines) of the TE0 (black) and TM0 (red) modes. The experimental dispersions are shown as squares. The corresponding bare waveguide modes are represented with dashed lines, and the exciton energies with horizontal dotted lines.

The resulting dielectric susceptibility appears to be a very rough approximation of the experimental one since the obtained complex susceptibility fails to account for three important features: (i) the amplitude of the peak in $\varepsilon_{1}$ near the exciton energy is underestimated; (ii) the strong dispersion of $\varepsilon_{1}$ below the bandgap, in the $3.0-3.3 \mathrm{eV}$ spectral range, is not reproduced; (iii) the very strong above-bandgap absorption of $\mathrm{GaN}$, where $\varepsilon_{2}$ reaches a value of about 2 , is absent in the model. These three features are strongly related since the steplike increase of $\varepsilon_{2}$ 
translates in a strong peak and a long low energy tail for $\varepsilon_{1}$ through Kramers-Kronig relations.

\section{Waveguide modeling with the Elliott-Tanguy dielectric susceptibility (model $C$ )}

This discrepancy was analytically solved by C. Tanguy, based on the Elliott model of the absorption of excitons and their diffusion states [31], in two different cases: the 3D exciton in a bulk semiconductor [32], as in the present work, and the 2D exciton in a quantum well [51], as in most polaritonic studies. Let us first emphasize that the simple band-to-band absorption, with a $\left(\sqrt{E-E_{G}}\right) / E$ dependence in the case of a $3 \mathrm{D}$ electron and hole density of states [orange curve for $\varepsilon_{2}$ in Fig. 4(b)], underestimates the experimental absorption near the band edge; this evidences the importance of Sommerfeld corrections related to the electron-hole plasma absorption edge, as calculated by Elliott [31]. The corresponding $\varepsilon_{2}$ is presented in Figs. 5(a) and 5(b) (dark green curve), with a homogeneous broadening $(20 \mathrm{meV}$ at $T=300 \mathrm{~K})$ and an inhomogeneous broadening $\sigma=10 \mathrm{meV}$. It reproduces the Heaviside-like absorption edge, plus the contribution of the excitons as a broadened peak when Coulomb interaction is taken into account. Following the work of C. Tanguy and accounting for the same inhomogeneous broadening, we derive the dispersion of $\varepsilon_{1}$. No additional constant is required for this approach and good agreement with the experimental spectroscopic ellipsometry is obtained below the bandgap up to the band edge; a slight discrepancy is observed above the bandgap, since $\varepsilon_{2}$ is still slightly underestimated.

A major interest of the so-called "Elliott-Tanguy" model presented in Figs. 5(a) and 5(b) is the possibility to calculate the complex dielectric susceptibility in the presence (dark green curve) and in the absence of Coulomb interaction (light green curve), in the limit of a vanishing Rydberg energy for the exciton as discussed in Ref. [32]. This last curve presents a marked peak at the band edge at $3.45 \mathrm{eV}$ that is solely related to the band-to-band absorption of $\mathrm{GaN}$ (no excitons) [52].

This susceptibility is used to calculate the dispersion of the bare photon modes of the waveguide within the same CamFR simulation as in the previous section. The corresponding calculated dispersions for the polariton modes are presented in Fig. 5. In order to properly fit the experimental dispersions of all TE modes, the oscillator strength of the $A, B$ excitons has been weighted by a factor of 0.95 , and the strength of the UV pole at $7 \mathrm{eV}$ has been slightly increased by a factor of 1.04 . The GaN thickness is adjusted to $e_{\mathrm{GaN}}=96 \mathrm{~nm}$, very close from the actual thickness $(100 \mathrm{~nm})$ measured on SEM images. The fit of the polariton dispersions is correct for the modes confined in $\mathrm{GaN}$ (TE0) as well as in the $(\mathrm{Al}, \mathrm{Ga}) \mathrm{N}$ cladding layer (TE1), thus showing that the refractive index and the waveguide thickness are independently determined. The
Model $C$ : Elliott-Tanguy exciton susceptibility
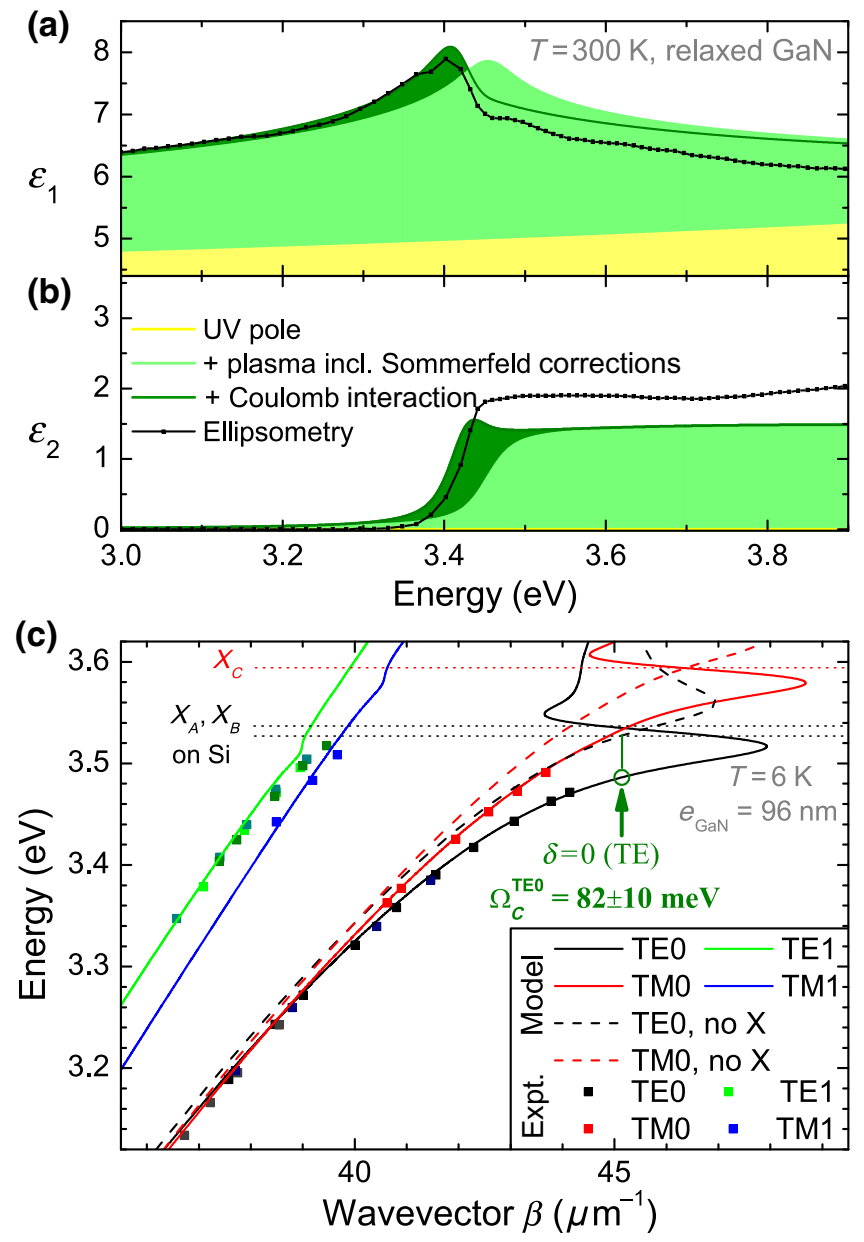

FIG. 5. Model $C$ based on the Elliott-Tanguy susceptibility. (a) Real part and (b) imaginary part of the GaN dielectric susceptibility (in-plane light polarization, TE mode, $T=300 \mathrm{~K}$ ): black square, solid lines represent ellipsometry measurements; Elliott-Tanguy model (including the same deep UV pole as in Fig. 4) with (dark green) and without (light green) Coulomb interaction. (c) Calculated dispersions (solid lines) of the TE0 (black), TM0 (red), TE1 (green), and TM1 (blue) eigenmodes based on the Elliott-Tanguy model of the dielectric susceptibility, and compared to the experimental dispersions (squares). The corresponding bare waveguide modes are represented with dashed lines, and the exciton energies with horizontal dotted lines.

mode profiles are shown in Appendix B. Note that, even in the absence of excitons, $\varepsilon_{1}$ displays a marked peak near the band edge, which leads to a strong bending of the dispersion of the bare waveguide modes. The zerodetuning condition $(\delta=0 \mathrm{meV})$ is therefore realized at a wavevector larger than that of the coupled oscillator model, $\beta_{\delta 0}^{C}=45.1 \pm 0.5 \mu \mathrm{m}^{-1}$ instead of $\beta_{\delta 0}^{A}=43.3 \pm$ $1 \mu \mathrm{m}^{-1}$, leading to a smaller value of the exciton-photon coupling $\Omega_{C}^{\mathrm{TE} 0}=82 \pm 10 \mathrm{meV}$. The uncertainty is estimated by varying the background refractive index, the 
exciton oscillator strength, and the GaN thickness in the fitting procedure.

\section{Empirical dielectric susceptibility from ellipsometry (model $D)$}

We have already highlighted that the extraction (or derivation) of a dielectric susceptibility of the $\mathrm{GaN}$ layer in the absence of excitons is a critical input for the calculation of the dispersion of the bare photon modes and, as a consequence, for the deduction of the exciton-photon coupling strength that relates the semiclassical approach to the Rabi splitting obtained in quantum theory. We propose here another approach where we assume that the susceptibility measured by spectroscopic ellipsometry [Figs. 6(a) and $6(\mathrm{~b})$, black line, $T=300 \mathrm{~K}]$ is accounting reliably for the excitonic transitions as well as for all other contributions to the background dielectric response of the $\mathrm{GaN}$ layer. Starting from the experimental susceptibility, the contribution of excitons to the dielectric constant is mathematically subtracted from the experimental data by considering excitons as classical harmonic oscillators. Only $A$ and $B$ excitons in their fundamental $(n=1)$ and excited states (up to $n=4$ ) are considered. An overall broadening of 23 $\mathrm{meV}$ is chosen for an optimal substraction of the excitonic contribution to the whole dielectric constant. The energies of $A$ and $B$ excitons are adjusted to fit the absorption front of $\varepsilon_{2}$ located just below the bandgap energy (3432 $\mathrm{meV}$ at $300 \mathrm{~K}$ ). With all the excitonic parameters being determined through this procedure, the corresponding contribution of the exciton to $\varepsilon_{1}$ is subtracted from the experimental data. Finally, the complex dielectric constant without the excitonic contributions is deduced initially at room temperature, and it is then obtained at all temperatures by shifting the energies, following the temperature dependence of the bandgap. The susceptibility at $300 \mathrm{~K}$ is presented in Figs. 6(a) and 6(b). This approach provides a third estimate of the GaN susceptibility in the absence of excitons.

The corresponding polariton dispersions are presented in Fig. 6. The main change compared to the Elliott-Tanguy model concerns the red shift of the band-edge resonance for the TE0 mode in the absence of excitons. This leads to an even larger wavevector for the zero-detuning condition $\left(\beta_{\delta 0}^{D}=45.8 \pm 0.5 \mu \mathrm{m}^{-1}\right)$, and a much smaller Rabi splitting $\Omega_{D}^{\mathrm{TE} 0}=55 \pm 6 \mathrm{meV}$.

\section{E. Comparison of the four models}

From the above discussion, the choice of the proper model for the dielectric susceptibility of the GaN active layer without excitons has a major impact on the determination of the exciton-photon coupling strength. The deduction of a single figure of merit of the strong-coupling regime - the Rabi splitting corresponding to the coupled oscillator quantum theory-is hiding a large part of the complexity of the electronic excitations in the active layer,
Model $D$ : Exciton susceptibility from ellipsometry
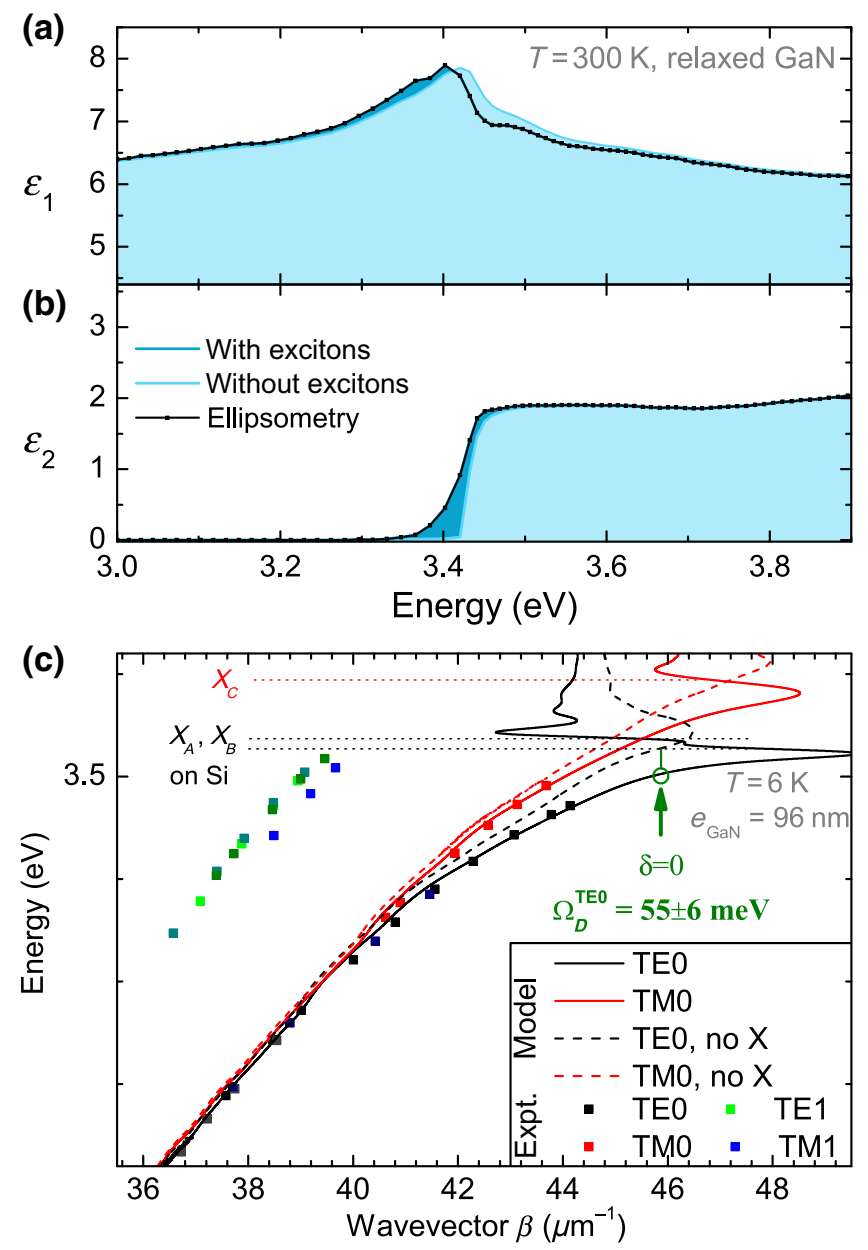

FIG. 6. Model $D$ based on the empirical susceptibility. (a) Real part and (b) imaginary part of the GaN dielectric susceptibility (in-plane light polarization, TE mode, $T=300 \mathrm{~K}$ ): black square, solid lines represent ellipsometry measurements; susceptibility without excitons (light blue) as the substraction of the experimental measurement and the Lorentzian oscillators. (c) Calculated polariton dispersions (solid lines) of the TE0 (black) and TM0 (red) modes, compared to the experimental dispersions (squares). The corresponding bare waveguide modes are represented with dashed lines, and the exciton energies with horizontal dotted lines.

and of the related dielectric susceptibility. From a practical point of view, good quantitative agreement between the experimental and simulated dispersions is not sufficient to secure a reliable estimation of the Rabi splitting. This is due to (i) the large oscillator strength of optical transitions in $\mathrm{GaN}$, which leads to a strong absorption from the excitons as well as from the unbound electron-hole pairs (a constant background value for $\varepsilon_{1}$ is too rough an approximation); and (ii) the polariton Rabi splitting being larger than the exciton binding energy, with the peak observed in the dielectric susceptibility in the absence of excitons lying in the same energy range as the bare photon mode at 
the zero-detuning condition. The simultaneous fulfilment of these two conditions is not specific to bulk GaN polariton devices and should also apply to $\mathrm{ZnO},(\mathrm{In}, \mathrm{Ga}) \mathrm{N} / \mathrm{GaN}$ quantum wells, and to some perovskites, transition metal dichalcogenides, and organic materials commonly used as active layers in room-temperature polaritonic devices.

Within the four proposed calculation schemes, the coupled oscillator model $(A)$ clearly overestimates the coupling strength; model $B$ with an almost constant group velocity for the bare photon mode and a single $X_{1 s}$ Lorentzian oscillator is not able to fit the whole dispersion and slightly overestimates the coupling strength. The empirical susceptibility based on ellipsometry (model $D$ ) provides the most conservative estimate of the Rabi splitting (55 $\pm 6 \mathrm{meV})$; finally, the Elliott-Tanguy model $(C)$ proposes a fully analytical expression for the susceptibility and leads to a intermediate value of $82 \pm 10 \mathrm{meV}$. We shall discuss here the discrepancy between models $B$, $C$, and $D$, which provide incompatible values of the coupling strength: it is known from Ref. [41] that, for a given set of exciton and photon energies, the splitting between transmission dips in a microcavity strongly depends on the value of the damping parameters for the photon mode (i.e., the mirror reflectivity) and the exciton mode (i.e., the exciton homogeneous broadening). Here these two parameters are part of the modeled dielectric susceptibilities through the absorption of the continuum (controlling the lifetime of the bare photon) and the broadening of the excitonic oscillators. The damping of the bare photon mode strongly varies among the models $B, C$, and $D$. Our attempt to perform reverse engineering of the dielectric susceptibility in the absence of excitons is based on two very different sets of assumptions in models $B-D$, leading to very different values of the wavevector $\beta_{\delta 0}$ at the zero-detuning condition, and of the splitting between the bare photon mode and the polariton mode at the zero-detuning condition. The discrepancy can therefore not be resolved. While all models do clearly assess the realization of the strongcoupling regime in the investigated GaN bulk waveguides, the actual value of the Rabi splitting is strongly model dependent; as a single physical quantity, it cannot hold for all the features of the coupling of the optical modes to the excitonic active layer in a regime where the Rabi splitting is larger than the excitonic binding energy, and where the exciton oscillator strength cannot be considered as a perturbation of the dielectric response of the active layer (in particular for $\varepsilon_{2}$ ).

\section{GENERAL CONSIDERATIONS ON THE ANISOTROPIC OPTICAL CHARACTER OF THE GaN ACTIVE REGION: TE AND TM POLARITON MODES}

The previous calculations were performed as if the $\mathrm{GaN}$ bulk material were isotropic. As detailed in Appendix A,
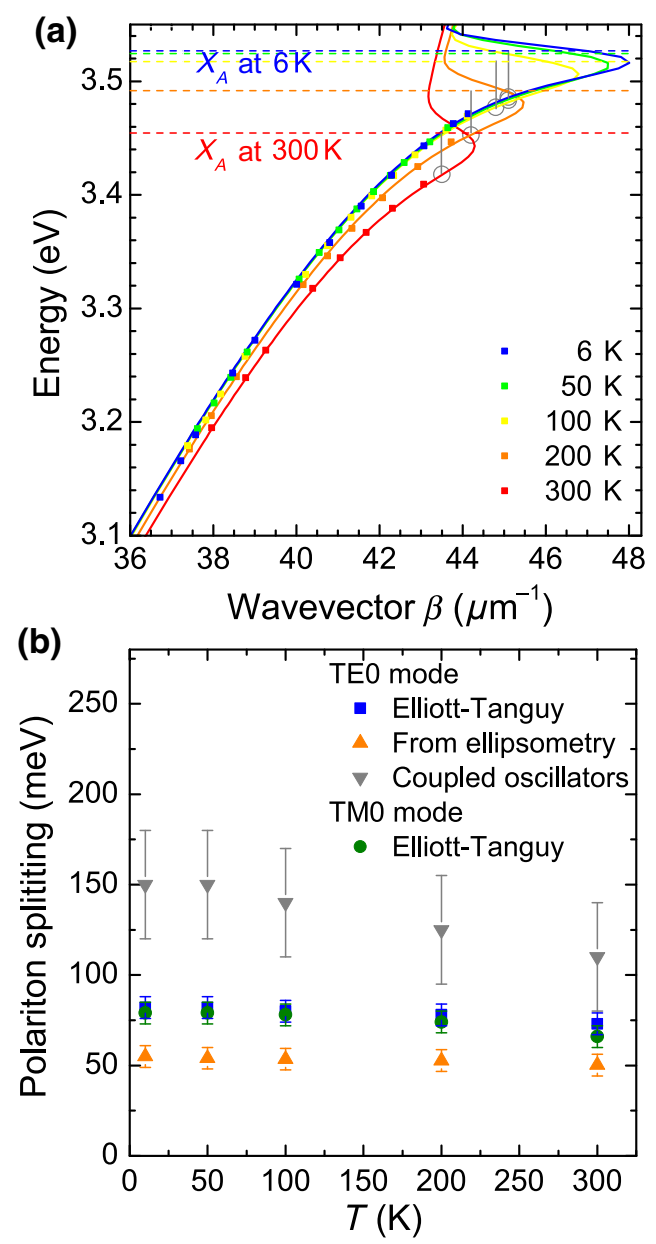

FIG. 7. (a) Experimental dispersions (filled squares) of the TE0 eigenmodes as a function of the sample temperature. The fit by the Elliott-Tanguy model is shown as solid lines with the same colors. The energies of the $X_{A}$ exciton are plotted with dashed horizontal lines; the gray open circles indicate the zero-detuning condition for each temperature, and the gray vertical segments represent the exciton-photon coupling strength $\Omega_{R} / 2$. (b) The corresponding values of $\Omega_{R}$ are presented for the TE0 and TM0 modes (blue and green symbols), as well as the fitted value for the coupled oscillator model (gray) and the empirical dielectric susceptibility (orange).

in the case of a GaN waveguide grown along the $c$ axis and taking into account the estimated strain of the GaNon-Si layer, pure selection rules (i.e., without mixing) are almost perfectly fulfilled: within a $1 \%$ accuracy $A$ and $B$ excitons interact with the TE mode only and the $C$ exciton interacts with the TM mode only. The calculation of the waveguide modes in TE and TM polarizations can be thus decoupled [53]. The TE mode depends only on the in-plane diagonal element of the dielectric tensor $\varepsilon_{\|}$and the calculation is strictly the same as in the isotropic case. The propagation equation related to the TM mode depends on the out-of-plane diagonal element of the dielectric tensor $\varepsilon_{\perp}$ and on the ratio $\varepsilon_{\|} / \varepsilon_{\perp}$. Indeed, the continuity of 

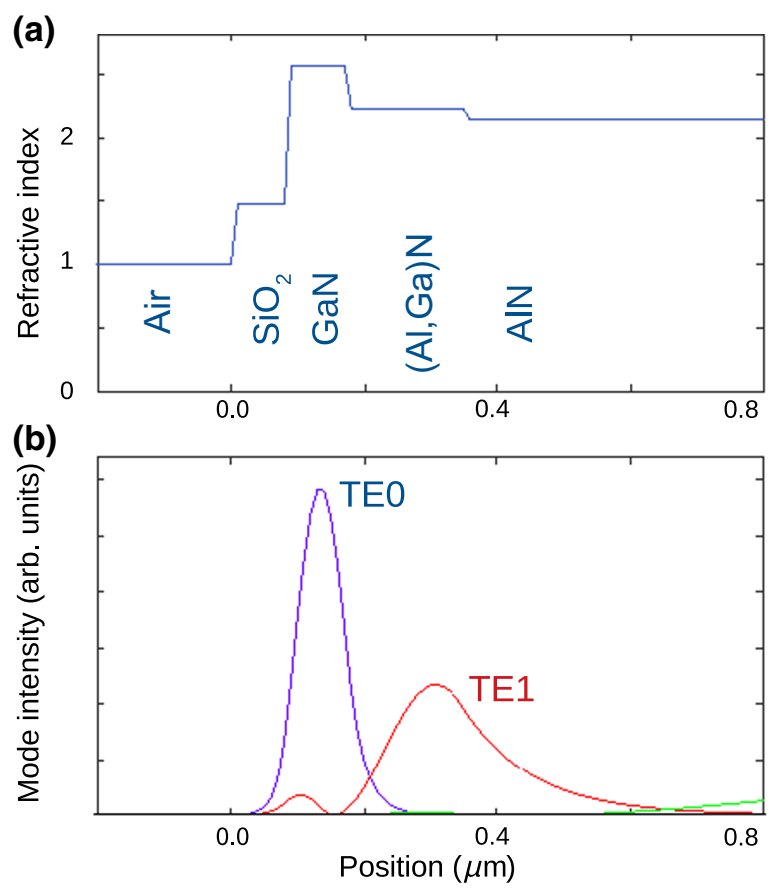

FIG. 8. Profiles of the (a) refractive index and (b) intensity of the first two TE modes (model $C, T=6 \mathrm{~K}, E=3.487 \mathrm{eV}$ ).

the magnetic fields at the interfaces also involves $\varepsilon_{\|}$. Without considering the excitonic resonances that are connected with optical selection rules, the ratio $\varepsilon_{\|} / \varepsilon_{\perp}$ is chosen in accordance with Ref. [49].

The deduced Rabi splitting for the TM0 mode based on the Elliott-Tanguy susceptibility (model $C$ ) and the empirical dielectric susceptibility (model $D$ ) are respectively worth 79 and $56 \mathrm{meV}$. Note that they are almost equal to those of the TE0 mode, which is consistent with the fact that the sum of $A$ and $B$ exciton oscillator strengths equals that of the $C$ exciton.

\section{TEMPERATURE DEPENDENCE OF THE RABI COUPLING}

The experimental polariton dispersions have been measured as a function of the sample temperature from low up to $T=300 \mathrm{~K}$, as shown with filled squares in Fig. 7(a). All dispersions are very similar, even though the exciton energies (horizontal dashed lines) shift to the red as the temperature is increased [54]. The fit by the ElliottTanguy model is plotted with solid lines: the homogeneous linewidth is increased from 5 to $20 \mathrm{meV}$ from $T=6$ to $300 \mathrm{~K},[30,55]$ while the weight of the exciton contribution to the susceptibility is decreased from 0.95 to 0.75 to properly reproduce the measured dispersions; in order to properly fit the photonic part of the dispersions at low wavevectors, it is also required to modify the weight of the Sellmeier deep UV pole by about $10 \%$ from low to room temperature. All other fitting parameters are kept constant across this set of temperature-dependent modeling.

The corresponding values of the Rabi splitting for the waveguide polaritons are presented in Fig. 7(b). We include the values deduced from the coupled oscillator model, the Elliott-Tanguy model, and the empirical model. The Rabi splitting decreases by about $11 \%$ for the TE0 mode, from 82 to $73 \mathrm{meV}$ for the Elliott-Tanguy case, and by $16 \%$ for the TM0 mode, revealing that the strongcoupling regime is maintained up to room temperature. Within the ellipsometry-based model $D$, the decrease of the Rabi splitting is also small (typically 5\%). Overall, all the models assess the robustness of the strong-coupling regime up to room temperature.

\section{CONCLUSION}

The dispersion of polaritons propagating in a bulk $\mathrm{GaN}$ waveguide is measured from $6 \mathrm{~K}$ to room temperature, and exhibits a strong negative dispersion coefficient that is a signature of the strong-coupling regime.

The analysis of the experimental dispersions is performed through four models of the exciton-photon coupling corresponding to quantum theory (model $A$ ) and semiclassical theory (models $B$ - $D$ ), assuming various dielectric susceptibilities for the active layer. We introduce two elaborate models leading to estimates of the excitonphoton Rabi splitting for TE0 modes of $55 \pm 6 \mathrm{meV}$ in an empirical approach based on ellipsometry measurements, and $82 \pm 10 \mathrm{meV}$ in the analytical model, the so-called "Elliott-Tanguy" model. We show that, whereas all models can quantitatively fit the experimental dispersions, they result in very different values of the exciton-photon coupling strengths. This complexity is related to two properties of the GaN active layer: the exciton binding energy is smaller than the coupling strength, and the background dielectric constant cannot be considered as frequency independent, even at large negative detunings, due to the large absorption above the bandgap. It appears that these two features are common to many materials chosen for their robust strong coupling up to room temperature. A strong bending of the dispersion in such polaritonic waveguides is not definitive proof of the strong-coupling regime. Interestingly, the proposed Elliott-Tanguy model can be adapted to polaritonic devices embedding such active layers with a large oscillator strength, including $\mathrm{ZnO},(\mathrm{In}, \mathrm{Ga}) \mathrm{N} / \mathrm{GaN}$ quantum wells, and some perovskites, transition metal dichalcogenides, and organic materials, therefore revisiting the determination of the Rabi splitting in those systems.

\section{ACKNOWLEDGMENTS}

The authors acknowledge funding from the French National Research Agency (Grants No. ANR-15-CE300020-02 and No. ANR-11-LABX-0014). 


\section{APPENDIX A: EXCITON ENERGIES AND BROADENINGS}

Let us discuss the chosen exciton energy levels of the bulk GaN active layer, which are the same for all the four models. Because of polarization-dependent selection rules, the TE0 mode is coupled to the $A$ and $B$ excitons. The energy of exciton $A$ is known from a reflectivity experiment performed on a GaN-on-Si sample with a similar thickness: $E_{X A}=3.527 \mathrm{eV}$. If we assume a biaxial strain for the $\mathrm{GaN}$ layer, this corresponds to a deformation $\varepsilon=$ $-1.3 \%$, leading to a $B$ exciton energy $E_{X B}=3.537 \mathrm{eV}$ and a $C$ exciton energy $E_{X C}=3.594 \mathrm{eV}[45,56]$. It should be mentioned that this deformation induces almost pure polarization selection rules, since the $C$ exciton transfers only $1.1 \%$ of its mainly $x$ polarized (along the growth axis, i.e., TM) oscillator strength to the $y$ and $z$ polarizations (in plane, i.e., TE), whereas the $A$ and $B$ excitons transfer the corresponding oscillator strength from the $x$ and $y$ polarizations to the $z$ polarization. This small deviation from purely polarized $A, B, C$ excitonic transitions is not included in the presented coupled oscillator model, but is accounted for in Sec. IV C.

The comparison between the experimental and model dielectric susceptibilities are based on room-temperature spectroscopic ellipsometry and reflectivity experiments performed on a series of thick GaN epilayers grown on silicon substrates, following the procedure detailed in Refs. [42,43].

The dielectric susceptibility presented in Figs. $4-6$ is measured and modeled at $T=300 \mathrm{~K}$ while the polariton dispersion is measured at $T=6 \mathrm{~K}$. Thus, to translate our modeling down to low temperature, the temperature dependence of the GaN refractive index has been taken into account through the Varshni law for energy shifts, and a temperature-dependent homogeneous linewidth for the excitonic states has been considered $(5 \mathrm{meV}$ at $T=6 \mathrm{~K})$.

\section{APPENDIX B: MODE PROFILES}

The dielectric structure of the GaN waveguide and the calculated eigenmodes is presented in Fig. 8 for an optical wave propagating at the energy corresponding to the zero-detuning condition of Fig. 5 (model $C, T=6 \mathrm{~K}$, $E=3.487 \mathrm{eV}$ ). The TE0 mode is mostly confined in the $\mathrm{GaN}$ active layer (84\%), whereas the TE1 mode is mostly guided in the cladding layers $[4 \%$ in $\mathrm{GaN}, 57 \%$ in $(\mathrm{Al}, \mathrm{Ga}) \mathrm{N}$, and $35 \%$ in $\mathrm{AlN}]$.

[1] A. Baas, J.-P. Karr, M. Romanelli, A. Bramati, and E. Giacobino, Optical bistability in semiconductor microcavities in the nondegenerate parametric oscillation regime: Analogy with the optical parametric oscillator, Phys. Rev. B 70, 161307 (2004).
[2] M. Vladimirova, S. Cronenberger, D. Scalbert, K. V. Kavokin, A. Miard, A. Lemaitre, J. Bloch, D. Solnyshkov, G. Malpuech, and A. V. Kavokin, Polariton-polariton interaction constants in microcavities, Phys. Rev. B. 82, 075301 (2010).

[3] J. Kasprzak, M. Richard, S. Kundermann, A. Baas, P. Jeambrun, J. M. J. Keeling, F. M. Marchetti, M. H. Szymanska, R. André, J. L. Staehli, V. Savona, P. B. Littlewood, B. Deveaud, and L. S. Dang, Bose-Einstein condensation of exciton polaritons, Nature (London) 443, 409 (2006).

[4] K. G. Lagoudakis, T. Ostatnicky, A. V. Kavokin, Y. G. Rubo, R. Andre, and B. Deveaud-Pledran, Observation of half-quantum vortices in an exciton-polariton condensate, Science 326, 974 (2009).

[5] K. G. Lagoudakis, B. Pietka, M. Wouters, R. André, and B. Deveaud-Plédran, Coherent Oscillations in an ExcitonPolariton Josephson Junction, Phys. Rev. Lett. 105, 120403 (2010).

[6] A. Amo, S. Pigeon, D. Sanvitto, V. G. Sala, R. Hivet, I. Carusotto, F. Pisanello, G. Leménager, R. Houdré, E. Giacobino, C. Ciuti, and A. Bramati, Polariton superfluids reveal quantum hydrodynamic solitons, Science 332, 1167 (2011).

[7] A. Kavokin, J. Baumberg, G. Malpuech, and F. Laussy, Microcavities (Oxford Science Publications, Oxford, UK, 2006).

[8] A. Amo and J. Bloch, Exciton-polaritons in lattices: A nonlinear photonic simulator, Comptes Rendus Physique 17, 934 (2016), polariton physics / Physique des polaritons.

[9] E. Wertz, L. Ferrier, D. D. Solnyshkov, R. Johne, D. Sanvitto, A. Lemaitre, I. Sagnes, R. Grousson, A. V. Kavokin, P. Senellart, G. Malpuech, and J. Bloch, Spontaneous formation and optical manipulation of extended polariton condensates, Nat Phys 6, 860 (2010).

[10] T. Gao, P. S. Eldridge, T. C. H. Liew, S. I. Tsintzos, G. Stavrinidis, G. Deligeorgis, Z. Hatzopoulos, and P. G. Savvidis, Polariton condensate transistor switch, Phys. Rev. B 85, 235102 (2012).

[11] P. Cristofolini, A. Dreismann, G. Christmann, G. Franchetti, N. G. Berloff, P. Tsotsis, Z. Hatzopoulos, P. G. Savvidis, and J. J. Baumberg, Optical Superfluid Phase Transitions and Trapping of Polariton Condensates, Phys. Rev. Lett. 110, 186403 (2013).

[12] C. Symonds, A. Lemaitre, E. Homeyer, J. C. Plenet, and J. Bellessa, Emission of Tamm plasmon/exciton polaritons, Appl. Phys. Lett. 95, 151114 (2009).

[13] M. Kaliteevski, S. Brand, R. A. Abram, I. Iorsh, A. V. Kavokin, and I. A. Shelykh, Hybrid states of Tamm plasmons and exciton polaritons, Appl. Phys. Lett. 95, 251108 (2009).

[14] M. Liscidini, D. Gerace, D. Sanvitto, and Daniele Bajoni, Guided Bloch surface wave polaritons, Appl. Phys. Lett. 98, 121118 (2011).

[15] L. K. van Vugt, S. Rühle, P. Ravindran, H. C. Gerritsen, L. Kuipers, and D. Vanmaekelbergh, Exciton Polaritons Confined in a ZnO Nanowire Cavity, Phys. Rev. Lett. 97, 147401 (2006).

[16] C. Czekalla, C. Sturm, R. Schmidt-Grund, B. Cao, M. Lorenz, and M. Grundmann, Whispering gallery mode lasing in zinc oxide microwires, Appl. Phys. Lett. 92, 241102 (2008). 
[17] M. A. Zimmler, F. Capasso, S. Muller, and Carsten Ronning, Optically pumped nanowire lasers: Invited review, Semic. Sci. and Technol. 25, 024001 (2010).

[18] S. Zhang, W. Xie, H. Dong, L. Sun, Yanjing Ling, J. Lu, Y. Duan, W. Shen, X. Shen, and Z. Chen, Robust excitonpolariton effect in a $\mathrm{ZnO}$ whispering gallery microcavity at high temperature, Appl. Phys. Lett. 100, 101912 (2012).

[19] P. M. Walker, L. Tinkler, M. Durska, D. M. Whittaker, I. J. Luxmoore, B. Royall, D. N. Krizhanovskii, M. S. Skolnick, I. Farrer, and D. A. Ritchie, Exciton polaritons in semiconductor waveguides, Appl. Phys. Lett. 102, 012109 (2013).

[20] P. M. Walker, L. Tinkler, D. V. Skryabin, A. Yulin, B. Royall, I. Farrer, D. A. Ritchie, M. S. Skolnick, and D. N. Krizhanovskii, Ultra-low-power hybrid light-matter solitons, Nat. Commun. 6, 8317 (2015).

[21] P. M. Walker, L. Tinkler, B. Royall, D. V. Skryabin, I. Farrer, D. A. Ritchie, M. S. Skolnick, and D. N. Krizhanovskii, Dark Solitons in High Velocity Waveguide Polariton Fluids, Phys. Rev. Lett. 119, 097403 (2017).

[22] D. M. D. Paola, P. M. Walker, R. P. A. Emmanuele, A. V. Yulin, J. Ciers, Z. Zaidi, J. F. Carlin, N. Grandjean, I. Shelykh, M. S. Skolnick, R. Butté, and D. N. Krizhanovskii, Ultrafast-nonlinear ultraviolet pulse modulation in an alingan polariton waveguide operating up to room temperature, arXiv:2009.02059v1.

[23] T. N. Oder, J. Y. Lin, and H. X. Jiang, Propagation properties of light in AlGaN/GaN quantum-well waveguides, Appl. Phys. Lett. 79, 2511 (2001).

[24] D. D. Solnyshkov, H. Terças, and G. Malpuech, Optical amplifier based on guided polaritons in $\mathrm{GaN}$ and $\mathrm{ZnO}$, Appl. Phys. Lett. 105, 231102 (2014).

[25] J. Ciers, J. G. Roch, J. F. Carlin, G. Jacopin, R. Butté, and N. Grandjean, Propagating Polaritons in III-Nitride Slab Waveguides, Phys. Rev. Appl. 7, 034019 (2017).

[26] O. Jamadi, F. Reveret, P. Disseix, F. Medard, J. Leymarie, A. Moreau, D. Solnyshkov, C. Deparis, M. Leroux, E. Cambril, S. Bouchoule, J. Zuniga-Perez, and G. Malpuech, Edge-emitting polariton laser and amplifier based on a $\mathrm{ZnO}$ waveguide, Light: Science \& Applications 7, 82 (2018).

[27] T. Guillet and C. Brimont, Polariton condensates at room temperature, Comptes Rendus Physique 17, 946 (2016).

[28] S. Richter, T. Michalsky, L. Fricke, C. Sturm, H. Franke, M. Grundmann, and R. Schmidt-Grund, Maxwell consideration of polaritonic quasi-particle Hamiltonians in multilevel systems, Appl. Phys. Lett. 107, 231104 (2015).

[29] S. Faure, T. Guillet, P. Lefebvre, T. Bretagnon, and B. Gil, Comparison of strong coupling regimes in bulk GaAs, GaN, and $\mathrm{ZnO}$ semiconductor microcavities, Phys. Rev. B 78, 235323 (2008).

[30] F. Réveret, P. Disseix, J. Leymarie, A. Vasson, F. Semond, M. Leroux, and J. Massies, Modelling of strong coupling regime in bulk GaN microcavities using transfer matrix and quasiparticle formalisms, Solid. State. Commun. 150, 122 (2010).

[31] R. J. Elliott, Intensity of optical absorption by excitons, Phys. Rev. 108, 1384 (1957).

[32] C. Tanguy, Optical Dispersion by Wannier Excitons, Phys. Rev. Lett. 75, 4090 (1995).
[33] A. B. Djurišić, Y. Chan, and E. Herbert Li, Progress in the room-temperature optical functions of semiconductors, Materials Science and Engineering: R: Reports 38, 237 (2002).

[34] T. Guillet, C. Brimont, P. Valvin, B. Gil, T. Bretagnon, F. Médard, M. Mihailovic, J. Zuniga-Perez, M. Leroux, F. Semond, and S. Bouchoule, Laser emission with excitonic gain in a $\mathrm{ZnO}$ planar microcavity, Appl. Phys. Lett. 98, 211105 (2011).

[35] F. P. Payne and J. P. R. Lacey, A theoretical analysis of scattering loss from planar optical waveguides, Optical and Quantum Electronics 26, 977 (1994).

[36] J. Levrat, R. Butté, E. Feltin, J. F. Carlin, N. Grandjean, D. Solnyshkov, and G. Malpuech, Condensation phase diagram of cavity polaritons in GaN-based microcavities: Experiment and theory, Phys. Rev. B 81, 125305 (2010).

[37] J. Zuniga-Perez, E. Mallet, R. Hahe, M. J. Rashid, S. Bouchoule, C. Brimont, P. Disseix, J. Y. Duboz, G. Gommé, T. Guillet, O. Jamadi, X. Lafosse, M. Leroux, J. Leymarie, F. Li, F. Réveret, and F. Semond, Patterned silicon substrates: A common platform for room temperature $\mathrm{GaN}$ and $\mathrm{ZnO}$ polariton lasers, Appl. Phys. Lett. 104, 241113 (2014).

[38] O. Jamadi, F. Réveret, E. Mallet, P. Disseix, F. Médard, M. Mihailovic, D. Solnyshkov, G. Malpuech, J. Leymarie, X. Lafosse, S. Bouchoule, F. Li, M. Leroux, F. Semond, and J. Zuniga-Perez, Polariton condensation phase diagram in wide-band-gap planar microcavities: $\mathrm{GaN}$ versus $\mathrm{ZnO}$, Phys. Rev. B. 93, 115205 (2016).

[39] O. Jamadi, F. Réveret, D. Solnyshkov, P. Disseix, J. Leymarie, L. Mallet-Dida, C. Brimont, T. Guillet, X. Lafosse, S. Bouchoule, F. Semond, M. Leroux, J. Zuniga-Perez, and G. Malpuech, Competition between horizontal and vertical polariton lasing in planar microcavities, Phys. Rev. B. 99, 085304 (2019).

[40] F. Médard, J. Zuniga-Perez, P. Disseix, M. Mihailovic, J. Leymarie, A. Vasson, F. Semond, E. Frayssinet, J. C. Moreno, M. Leroux, S. Faure, and T. Guillet, Experimental observation of strong light-matter coupling in $\mathrm{ZnO}$ microcavities: Influence of large excitonic absorption, Phys. Rev. B 79, 125302 (2009).

[41] V. Savona, L. C. Andreani, P. Schwendimann, and A. Quattropani, Quantum well excitons in semiconductor microcavities: Unified treatment of weak and strong coupling regimes, Solid State Comm. 93, 733 (1995).

[42] N. Antoine-Vincent, F. Natali, M. Mihailovic, A. Vasson, J. Leymarie, P. Disseix, D. Byrne, F. Semond, and J. Massies, Determination of the refractive indices of AlN, GaN, and $\mathrm{Al}_{x} \mathrm{Ga}_{1-x} \mathrm{~N}$ grown on (111)Si substrates, J. Appl. Phys. 93, 5222 (2003).

[43] E. Mallet, F. Réveret, P. Disseix, T. V. Shubina, and J. Leymarie, Influence of excitonic oscillator strengths on the optical properties of gan and zno, Phys. Rev. B 90, 045204 (2014).

[44] P. Bienstman and R. Baets, Optical modelling of photonic crystals and VCSELs using eigenmode expansion and perfectly matched layers, Optical and Quantum Electronics 33, 327 (2001).

[45] M. Julier, J. Campo, B. Gil, J. P. Lascaray, and S. Nakamura, Determination of the spin-exchange interaction constant in wurtzite GaN, Phys. Rev. B 57, R6791 (1998). 
[46] R. Butté, G. Christmann, E. Feltin, J. F. Carlin, M. Mosca, M. Ilegems, and N. Grandjean, Room-temperature polariton luminescence from a bulk GaN microcavity, Phys. Rev. B 73, 033315 (2006).

[47] F. Réveret, I. R. Sellers, P. Disseix, J. Leymarie, A. Vasson, F. Semond, M. Leroux, and J. Massies, Strong coupling in bulk GaN microcavities grown on silicon, Phys. Status Solidi (C) 4, 108 (2007).

[48] F. Réveret, P. Disseix, J. Leymarie, A. Vasson, F. Semond, M. Leroux, and J. Massies, Influence of the mirrors on the strong coupling regime in planar GaN microcavities, Phys. Rev. B 77, 195303 (2008).

[49] S. Shokhovets, R. Goldhahn, G. Gobsch, S. Piekh, R. Lantier, A. Rizzi, V. Lebedev, and W. Richter, Determination of the anisotropic dielectric function for wurtzite AlN and $\mathrm{GaN}$ by spectroscopic ellipsometry, J. Appl. Phys. 94, 307 (2003).

[50] T. Kawashima, H. Yoshikawa, S. Adachi, S. Fuke, and K. Ohtsuka, Optical properties of hexagonal GaN, J. Appl. Phys. 82, 3528 (1997).

[51] C. Tanguy, P. Lefebvre, H. Mathieu, and R. J. Elliott, Analytical model for the refractive index in quantum wells derived from the complex dielectric constant of Wannier excitons in noninteger dimensions, J. Appl. Phys. 82, 798 (1997).

[52] S. Shokhovets, G. Gobsch, and O. Ambacher, Momentum matrix element and conduction band nonparabolicity in wurtzite GaN, Appl. Phys. Lett. 86, 161908 (2005).

[53] E. Rosencher and B. Vinter, Optoelectronics (Cambridge University Press, Cambridge, UK, 1998).

[54] D. Brunner, H. Angerer, E. Bustarret, F. Freudenberg, R. Hopler, R. Dimitrov, O. Ambacher, and M. Stutzmann, Optical constants of epitaxial AlGaN films and their temperature dependence, J. Appl. Phys. 82, 5090 (1997).

[55] A. Trichet, L. Sun, G. Pavlovic, N. A. Gippius, G. Malpuech, W. Xie, Z. Chen, M. Richard, and L. S. Dang, One-dimensional $\mathrm{ZnO}$ exciton polaritons with negligible thermal broadening at room temperature, Phys. Rev. B 83, 041302(R) (2011).

[56] A. Alemu, B. Gil, M. Julier, and S. Nakamura, Optical properties of wurtzite $\mathrm{GaN}$ epilayers grown on A-plane sapphire, Phys. Rev. B 57, 3761 (1998). 